\title{
Evaluating Bi-temporal Dynamics and Trend of Urbanization- induced Land Cover Temperature in Shanghai, China
}

Fei Liu $^{\mathrm{a},} *$, Yuji Murayama ${ }^{\mathrm{b}}$

${ }^{a}$ Graduate School of Life and Environmental Sciences, University of Tsukuba, 1-1-1 Tennodai, Tsukuba, 305-8572, Ibaraki, Japan, Email: rscugliufei@gmail.com

${ }^{b}$ Faculty of Life and Environmental Sciences, University of Tsukuba, 1-1-1 Tennodai, Tsukuba, 305-8572, Ibaraki, Japan; Email: mura@geoenv.tsukuba.ac.jp

* Corresponding author

Keywords: Land Cover Temperature, Urban Heat Island (UHI), Urban Thermal Environment

\begin{abstract}
:
In China today, massive land use/land cover change (LULCC) driven by urbanization has become a dominant phenomenon in the interactions between the human system and land surface. Drastic urbanization has changed land surface properties where a great deal of physical landscapes has been replaced by artificial buildings, and this evolution has brought about great potential risks and pressures physically and socially. In this study, taking the Shanghai metropolitan area (SMA) as the study area, we examine the spatiotemporal dynamics of temperature in urban land cover and explore the sustainable strategies for future development.
\end{abstract}

Shanghai, one of the Chinese gigantic cities, had the residential population of 24.15 million in 2015. The phenomena of urban heat island (UHI) in SMA have become prominent by the urbanization-related anthropic activities and increasing population. Until the present, a substantial number of investigations has been conducted in the Shanghai's thermal environment based on remote sensing data. However, the comprehensive assessments of urban thermal environment are not enough in Shanghai. This kind of research is expected to provide a significant paradigm for the exploration of a sustainable development of Chinese megacities, which examines the mechanism of the interactions between urban thermal environment and human system and addresses its adverse effect on the urban environment.

In this study, bi-temporal Landsat satellite data (Sep.2000 and Aug.2015) were used for the detection of LULCC and the estimation of land surface temperature (LST) in SMA. The LST was retrieved using the radiation transfer equation (RTE), and then normalized and graded in order to remove the influence of the seasonality and local climate characteristics. The spatiotemporal configuration and variation of urban thermal environment in SMA between 2000 and 2015 were synthetically monitored and evaluated through the analyses of the urban-rural gradient, gravity center change, spatial landscape pattern, and geographically weighted regression. Furthermore, the spatial determinants for the formation of UHI in SMA were identified in the analysis.

The results showed that (1) in 2000, the LST in the downtown of SMA was higher than that in the outskirts and rural area; (2) the extent of UHI in SMA was gradually expanding from 2000 to 2015 along the direction of urban growth, however, the UHI intensity in the downtown area was relatively declining due to the explosive increase of impervious surfaces in the suburban and rural area; (3) the spatiotemporal evolution of SMA's urban thermal environment was highly consistent with the track of LULCC, and greatly influenced by the spatial pattern and variation of urban landscape; (4) impervious surfaces obviously facilitated and strengthened the UHI effect, inversely, greenbelt and water space contributed to the mitigation and alleviation of the UHI effect; and (5) population convergence, industrial growth, dense land utilization, and urban development policies also affected the SMA's urban thermal environment. 